\title{
LA CLASSE INVERSÉE A L'ISSAE-CNAM DE L'UNIVERSITÉ LIBANAISE : ENTRE ACTION, RÉFLEXION ET MÉMORISATION
}

\author{
Stéphane- Ahmad HAFEZ, Université Libanaise, faculté de pédagogie, \\ stephanehafez@hotmail.com \\ Maha MROUEH, ISSAE-Cnam, mahamroueh@hotmail.com
}

$10.31902 /$ fll.29.2019.11

UDK 371.3:81'232]:378(569.3)

\begin{abstract}
Résumé : Cet article a pour objectif de rendre compte d'une expérimentation de la classe inversée en cours de langue à l'ISSAE-Cnam de l'Université Libanaise. Il s'agissait de réaliser trois macro-tâches : une capsule, une page HTML, un exposé via Google Slides, en suivant trois étapes: la phase découverte (à distance), la phase d'analyse et d'approfondissement (en présentiel et en groupe) et la phase de production, d'évaluation et de réajustement (en présentiel et à distance individuellement et en groupe). Les résultats sont concluants : les étudiants se sont engagés dans le projet. Ils ont pris conscience de leur manière d'apprendre et réalisé les tâches dans un esprit de partage et de collaboration. Ces quelques observations ne nous empêchent pas pourtant de déduire que la classe inversée est une arme à double tranchant : elle peut motiver des jeunes curieux de nouveautés, accros aux nouvelles technologies, mais elle peut être aussi une source d'ennui si elle est mal préparée et mal utilisée. En effet, l'investissement en énergie et en temps de la part des protagonistes n'est guère à négliger. Par conséquent, la classe conventionnelle ne peut se substituer par la classe inversée, c'est le bon équilibre entre ces deux approches qui fera toute la différence.
\end{abstract}

Mots-clés : classe inversée, expérimentation, collaboration, autonomie, mémorisation, mémoire perceptive.

Au cours de ces dernières années, la classe inversée suscite beaucoup I'intérêt des enseignants, notamment de FLE. Pourtant, cette approche qui se situe au carrefour de plusieurs approches pédagogiques (l'auto-apprentissage, l'apprentissage collaboratif, la pédagogie différenciée) n'est pas nouvelle. Dès 1990, Mazur, professeur à Harvard, proposait déjà l'idée de sortir l'enseignement magistral de sa classe. C'est par hasard que Sams et Bergmann, deux professeurs du Colorado, ont implanté cette approche qui allait être par la suite nommée le flip teaching (Roy, 1). En s'appuyant sur les nouvelles technologies, la classe inversée a su se réinventer pour conquérir d'autres types de publics.

Souhaitant apporter du nouveau à ses pratiques de classe et impliquer davantage ses apprenants dans leur processus d'apprentissage, Maha MROUEH, enseignante à l'Institut supérieur des sciences appliquées et économiques ${ }^{1}$ (IS-

\footnotetext{
${ }^{1}$ Pour avoir une idée de la situation du français à l'ISSAE, lire nos articles :

Français sur objectifs universitaires au Liban: dispositif, contenus, enquête de terrain
} 
SAE) de l'Université Libanaise (UL) et co-auteure de cet article a décidé d'appliquer la classe inversée. En tant que collègue et didacticien, nous l'avons accompagnée dans son projet.

Dans quelle mesure la classe inversée pourrait-elle donner un second souffle au cours de langue? S'agirait-il d'une alternative ou d'une complémentarité à la classe conventionnelle? Quelle démarche pour l'appliquer dans de bonnes conditions?

Pour répondre à notre questionnement, nous nous appuyons sur le bilan de notre expérimentation. Tout d'abord, nous définirons brièvement la classe inversée, les types et la démarche. Puis, nous présenterons les contenus, les étapes ainsi que les résultats de l'expérimentation. Enfin, nous rendrons compte du retour de l'enseignante et des étudiants sur la classe inversée.

\subsection{La classe inversée, en bref}

"Approche ", " méthode ", " activité ", " démarche ", " outil ", la classe inversée est connue sous différentes appellations, dont le dénominateur commun est l'inversion du concept traditionnel de la classe : le cours magistral cède la place au cours dialogué en classe et aux exercices à la maison. Nous rappelons avec Dufour « inverser la classe revient donc à profondément modifier le rôle traditionnel de l'enseignant. II passe du face-à-face au côteà-côte, permettant ainsi la mise en place d'une co-construction des savoirs. "Avec la classe inversée, " on est bien dans une stratégie de structuration des connaissances qui correspond mieux aux caractérsitiques des nouveaux élèves, surtout en milieu professionnel » P. Beriocchini et E. Constanzo, (P.67).

Dans La classe à l'envers pour apprendre à l'endroit, guide pratique pour débuter en classe inversée (Lecoq et Lebrun, 7), on en distingue trois types.

- Le premier type est le plus connu. Ce sont des leçons sous forme de vidéos à regarder à la maison ou à la bibliothèque et des exercices à faire en classe.

- Le deuxième type nommé la "classe translatée ", consiste en une recherche documentaire et des travaux préparatoires à la maison, des exposés, des débats, des activités de modélisation en classe. Ce type de classe inversée permet d" "ancrer les savoirs dans leur contexte et dans l'expérience concrète " et de "penser les apprentissages et les conditions dans lesquelles l'élève va apprendre ».

http://eprints.aidenligne-francais-universite.auf.org/642/1/Fran\%C3\%A7ais_sur_objectifs_universitaires_au_Liban_St\%C3\%A9phane_HAFEZ.pdf

Les outils numériques au service du fou : quelle approche pour des formations motivantes et interactives?

http://eprints.aidenligne-francais-universite.auf.org/791/1/FOU__Outils_num\%C3\%A9riques.pdf 
- Le troisième est une hybridation des deux autres et repose sur la construction d'un scénario des séquences d'apprentissage marqué par un mouvement en quatre temps. A distance, l'élève fait des travaux de recherche, puis on fait en classe des exposés qui entraînent la création à distance de textes et de vidéos qui sont ensuite source de débats en classe. Des trois types, c'est ce dernier type que nous adoptons dans le cadre de notre expérimentation.

La réussite de la classe inversée est soumise à des conditions. II s'agit de redéfinir le rôle des protagonistes (enseignant et apprenants), de disposer d'un environnement technopédagogique, de personnaliser l'approche en fonction de son milieu professionnel et des besoins de ses apprenants (L'agence des usages- réseau Canopé).

\subsection{La classe inversée et la mémorisation}

La classe inversée " aide à faire ", mais elle permet aussi de "réflechir » et de " mémoriser » (Taurisson et Herviou, 10). C'est une mémorisation active qui se déroule en trois étapes :

- L'encodage est l'étape pendant laquelle les apprenants prennent contact avec les documents déclencheurs. Ils sont capables de classer, de catégoriser l'information, mais aussi de présenter clairement les différents éléments, pour les expliquer et aider à leur mémorisation.

- La rétention, c'est le passage de l'information de la mémoire de travail vers la mémoire à long terme. Cette étape consiste à s'approprier l'information, en la manipulant par le biais d'activités variées qui prennent en considération les différents profils d'apprentissage. C'est la mise en application de l'information dans le vécu de l'apprenant. Elle utilise les éléments sémantiques (la signification associée à l'information) pour classer l'information afin de la stocker durablement.

- La récupération, c'est le passage de l'information de la mémoire à long terme à la mémoire de travail, dans un but de réutilisation et de transfert. Cette troisième étape est le moment où on se rappelle les souvenirs d'événements afin de les réutiliser (FMP Formation).

\section{L'expérimentation}

\subsection{Préparation du terrain}

Un mois avant le début de l'expérimentation, Mme MROUEH a revu le programme en vigueur et a élaboré le matériel en fonction des objectifs fixés. Elle a sélectionné des supports (des extraits de livres et d'émissions, des vidéos, des films documentaires...). Parallèlement à ces ressources externes, elle a conçu ses propres outils de travail : 
- des capsules ont été créées avec Powtoon, un logiciel qui permet de créer des vidéos animées. Ces documents servaient à susciter l'intérêt des apprenants à expliquer des notions complexes ou à résumer un cours ;

- des activités en ligne ont été élaborées avec LearningApps, un outil de création d'activités pédagogiques interactives;

- des grilles d'(auto)-évaluation et des tests ont été élaborés en version papier et sur Evalbox, une plate-forme d'auto-évaluation pour gérer des tests et des grilles d'évaluation en ligne.

- une batterie de petits exercices a été conçue pour constituer les groupes d'étudiants en fonction des différents styles d'apprentissage. L'ensemble de ces ressources a été hébergée sur Google Classroom, un espace d'échange et de partage virtuel.

En ce qui concerne les pratiques de classe, l'enseignante a tenté de favoriser le travail en autonomie, la gestion de groupe, du temps et de l'espace. Elle a instauré un climat de confiance et d'échange enseignants-apprenants et apprenants-apprenants. Lorsque les bons réflexes se sont mis en place, elle a expliqué sous forme d'activités le principe de la classe inversée. Le jour J, après avoir constitué les groupes en concertation avec les étudiants, elle a fait analyser la tâche langagière et a distribué une feuille de route. Celle-ci précisait le résultat attendu, la durée de l'activité, les modalités de travail et les adresses de sites à consulter.

\subsection{Contenus}

L'expérimentation a concerné trois macro-tâches d'un module de français sur objectif universitaire (FOU), de 30 h. Elle s'est étalée sur un semestre, d’octobre à février 2018.

\begin{tabular}{|l|l|l|l|}
\hline \multicolumn{2}{|l|}{ Unité didactique 1 } & Unité didactique 2 & \multicolumn{2}{l|}{ Unité didactique 3 } \\
\hline Domaine & $\begin{array}{l}\text { Economie-gestion } \\
\text { Niveau }\end{array}$ & B1+ & \multicolumn{2}{l|}{} \\
\hline $\begin{array}{l}\text { Tâche langa- } \\
\text { gière }\end{array}$ & $\begin{array}{l}\text { Présenter un phénomène } \\
\text { monétaire dans le cadre } \\
\text { d'un exposé via Google } \\
\text { Slides : I'inflation. }\end{array}$ & $\begin{array}{l}\text { Faire une capsule de 2 mn pour } \\
\text { expliquer un dispositif financier : le } \\
\text { microcrédit. }\end{array}$ & $\begin{array}{l}\text { Ecrire une page HTML sur l'évolution } \\
\text { des startups au Liban. }\end{array}$ \\
\hline $\begin{array}{l}\text { Objectifs } \\
\text { pragma- } \\
\text { tiques }\end{array}$ & $\begin{array}{l}\text { Définir, caractériser, donner } \\
\text { un exemple, nommer, citer. }\end{array}$ & $\begin{array}{l}\text { Définir, faire un petit rappel } \\
\text { historique, expliquer le } \\
\text { fonctionnement, donner des } \\
\text { précisions, énumérer, reformuler. }\end{array}$ & $\begin{array}{l}\text { Commenter des chiffres statistiques, } \\
\text { comparer, analyser, interpréter, } \\
\text { déduire, nuancer, conclure. }\end{array}$ \\
\hline $\begin{array}{l}\text { Objectifs lin- } \\
\text { guistiques }\end{array}$ & $\begin{array}{l}\text { Lexique de l'inflation } \\
\text { Tournures impersonnelles } \\
\text { Lronoms relatifs composés } \\
\text { La mise en relief }\end{array}$ & $\begin{array}{l}\text { Lexique du microcrédit } \\
\text { Verbes- adverbes } \\
\text { Connecteurs logiques }\end{array}$ & $\begin{array}{l}\text { Le lexique des startups/ de la } \\
\text { statistique } \\
\text { La comparaison, l'opposition }\end{array}$ \\
\hline
\end{tabular}




\begin{tabular}{|c|c|c|c|}
\hline \begin{tabular}{|l|} 
Objectifs \\
sociocultu- \\
rels / écono- \\
miques
\end{tabular} & L'inflation au Liban & $\begin{array}{l}\text { le système des microcrédits au } \\
\text { Liban }\end{array}$ & Les startups au Liban \\
\hline Durée & $\begin{array}{l}3 \mathrm{~h} \text { en présentiel } \\
1 \mathrm{~h} 30 \text { à distance }\end{array}$ & $\begin{array}{l}3 \text { h en présentiel } \\
1 \text { h } 30 \text { à distance }\end{array}$ & $\begin{array}{l}4 \mathrm{~h} 30 \mathrm{~h} \text { en présentiel } \\
2 \mathrm{~h} \text { à distance }\end{array}$ \\
\hline \begin{tabular}{|l|l} 
Activités \\
langagières
\end{tabular} & \multicolumn{3}{|c|}{ Réception orale/ écrite/ Production orale/ écrite } \\
\hline \begin{tabular}{|l|}
$\begin{array}{l}\text { Ressources } \\
\text { externes }\end{array}$ \\
\end{tabular} & $\begin{array}{l}\text { L'inflation, c'est quoi ? } \\
\text { https://www.youtube.com/ } \\
\text { watch?v=LUC2qdpPGDs } \\
\text { Définition } \\
\text { http://www.toupie.org/ } \\
\text { Dictionnaire/Inflation.htm } \\
\text { Exposé sur l'inflation : un } \\
\text { modèle } \\
\text { http://www.etudier.com/ } \\
\text { dissertations/Expose-Sur- } \\
\text { l'Inflation/57143.html }\end{array}$ & $\begin{array}{l}\text { Le microcrédit } \\
\text { https://www.youtube.com/ } \\
\text { watch?v=wgo3CxdG6EM\&t=35s } \\
\text { (1: } 55 \\
\text { Micro-crédit : qu'est-ce que c'est? } \\
\text { https://www.radins.com/dossiers/ } \\
\text { banque/micro-credit-qu-est-ce-que- } \\
\text { c-est,1236.html } \\
\text { Micro-crédit au Liban } \\
\text { https://www.microworld.org/ } \\
\text { fr/news-from-the-field/article/ } \\
\text { microfinance-au-liban } \\
\text { Micro-crédit : un nouveau moyen } \\
\text { pour financer son entreprise } \\
\text { http://www.journaldunet.com/ } \\
\text { economie/tendances/microcredit/ } \\
\text { index.shtml }\end{array}$ & $\begin{array}{l}\text { C'est quoi une startup ? } \\
\text { http://le-shift.co/c-est-quoi-une- } \\
\text { startup-definition-difference- } \\
\text { entreprise/ } \\
\text { Les } 10 \text { chiffres incontournables de } \\
\text { l'écosystème startups français } \\
\text { e-https://www.maddyness.com/ } \\
\text { entrepreneurs/2017/03/21/frenchtech- } \\
\text { ecosysteme-startup-francais-10- } \\
\text { chiffres/ } \\
\text { Exploiter un tableau statistique } \\
\text { http://www.i-manuel.fr/ } \\
\text { PASTMG_ECOT/PASTMG_- } \\
\text { ECOTpart11dos14CO2fm1. } \\
\text { htm?Mobile=1\&Source=\%2F_- } \\
\text { layouts\%2Fmobile\%2Fview. } \\
\text { aspx\%3FList\%3D8ab2c4d7- } \\
\text { 8d38-4c0d-b77c- } \\
\text { 1408659e6834\%26View\%3Da841ad66- } \\
\text { 8424-42d0-84ec- } \\
\text { 62b3a5bfde65\%26CurrentPage\%3D1 }\end{array}$ \\
\hline \begin{tabular}{l|l} 
Types \\
d'exercices
\end{tabular} & \multicolumn{3}{|c|}{$\begin{array}{l}\text { QCM, vrai/ faux, questions ouvertes- semi-ouvertes, résolution de problèmes, étude de cas, débats/ } \\
\text { discussions, jeu de rôle, exercices d'application, apprentissage par les pairs }\end{array}$} \\
\hline \begin{tabular}{|l|} 
Plate- \\
formes et \\
applications
\end{tabular} & \multicolumn{3}{|c|}{ Google Class, Google Slides, Googles Forms, Powtoon, Mentimeter, LearningApps, Padlet... } \\
\hline $\begin{array}{l}\text { Modalités } \\
\text { de travail }\end{array}$ & \multicolumn{3}{|c|}{ En présentiel, à distance, individuel et en groupe } \\
\hline
\end{tabular}

Tableau 1. Présentation générale des unités didactiques

\section{Moments-clés}

Indépendamment des macro-tâches, la classe inversée s'est déroulée en trois étapes.

\subsection{Avant le cours : phase découverte}

Les étudiants étaient invités à regarder des capsules ou des vidéos à distance et à répondre à un questionnaire en ligne conçu avec Google Forms 
(thème, situation de communication, etc). Puis ils devaient donner la définition du concept sous forme de points sur Padlet, un mur virtuel et interactif.

\subsection{Pendant le cours : phase d'analyse et d'approfondissement}

L'enseignante vérifiait si les étudiants avaient regardé la vidéo en leur demandant de restituer oralement les informations. Pendant ce temps, elle notait au tableau le nouveau lexique, les idées essentielles et guidait les apprenants en difficulté à reformuler leurs réponses. Selon les cas, elle leur proposait des exercices plus complexes ou passait directement à l'étape d'analyse de corpus. Ce travail de systématisation a été complété par des exercices à faire à distance.

\subsection{Après le cours : phase de production, d'évaluation et de réajustement}

Les petits groupes réalisaient et mettaient en ligne leurs projets sous $48 \mathrm{~h}$. Le jour de présentation du produit final, l'ensemble de la classe munie d'une grille d'évaluation attribuait des notes, selon des critères fixés auparavant. Ces séances de restitution étaient toujours basées sur l'écoute, l'échange et donnaient l'occasion aux étudiants d'apprécier le travail de l'autre.

\section{Résultats de l'expérimentation}

Notre étude est qualitative et ne prétend pas à l'exhaustivité. Les résultats reflètent un seul aspect de la classe inversée appliquée dans un contexte bien déterminé. D’autres études suivront pour affiner nos données.

L'analyse du produit final révèle que les étudiants ont réussi majoritairement leurs tâches. Les capsules qu'ils ont créées pour expliquer l'inflation étaient l'oeuvre de professionnels. D'ailleurs, elles ont fait le tour de l'ISSAE. L'exposé via Google Slides a également eu du succès. Les exposés ont été filmés et postés sur Google Class. Ils ont fait l'objet d'un vote en ligne. Quant à la page HTML, elle a été écrite à plusieurs mains via Googles Docs. Certains textes étaient mal rédigés ce qui a nécessité un travail de réécriture. Par conséquent, cette tâche a pris plus de temps que prévu.

Ces bons résultats se justifient par le plan d'action longuement réfléchi et le bon dosage entre le travail à réaliser en présentiel et à distance. A cela s'ajoutent d'autres facteurs comme l'accessibilité des applications, le respect des étapes qui faisiaent écho avec celles de la mémorisation et surtout la répartition des groupes.

Dans la mesure du possible, les styles d'apprentissage de Kolb (1984) étaient représentés dans chaque groupe. L'objectif était de favoriser la collaboration et l'apprentissage constructiviste. En général, l'étudiant ayant un style d'apprentissage accommodateur (apprentissage par l'essai-erreur et les 
manipulations) a collaboré avec le camarade dont le style d'apprentissage est assimilateur (apprentissage par la réflexion et la théorisation), celui divergent (apprentissage par l'expérience) ou encore convergent (apprentissage par la résolution de problème et les applications pratiques d'idées).

Cette constitution des groupes n'aurait pas abouti si l'ambiance de la classe n'y avait pas été propice. Dès le début, la salle de cours est devenue un lieu de travail où régnaient la franchise, la transparence et l'humour. Cette ambiance a donné envie à un grand nombre d'étudiants de s'impliquer dans leur apprentissage car travailler n'était plus lassant pour eux. Ils se concertaient, discutaient, réfléchissaient, produisaient... Comme on le sait, l'autorité constante et rigide ne plaît pas à la nouvelle génération d'étudiants, qui est à la recherche d'un enseignant-coach qui lui permet de mieux s'épanouir, tout en développant ses compétences.

Bien que l'expérimentation de la classe inversée ait globalement réussi, elle présente des points faibles qui gagneraient à être améliorés dans les formations à venir :

- L'excès de matériel : le dispositif était riche de supports et d'activités que l'enseignante n'a pas eu le temps d'exploiter tous. II serait judicieux de les classer par ordre d'importance et d'intérêt. Ces documents supplémentaires serviraient à occuper les étudiants plus rapides que d'autres.

- Le non respect du calendrier et le bruit : il arrivait à l'enseignante de fermer les yeux sur les étudiants retardataires, paresseux ou bruyants. Ils n'étaient pas nombreux mais ils ont failli porter préjudice à l'expérimentation. Heureusement, l'enseignante s'est rattrapée au bon moment. Elle les a rappelés à l'ordre. Le contrat d'apprentissage devrait rester la référence en la matière notamment pour gérer le bruit occasionné par le travail de groupe.

- L'absence de récompense : les bonnes notes étaient le seul moyen pour évaluer les étudiants. II conviendrait de proposer un système de récompense pour motiver les étudiants et assurer la visibilité de tels projets.

\section{Retour des protagonistes sur la classe inversée}

A l'issue de l'expérimentation l'enseignante et les étudiants ont fait part de leurs réflexions à travers un questionnaire et des groupes de discussion. Nous en présentons les résultats les plus pertinents avec de courts extraits illustrant leurs propos.

\subsection{Les étudiants}

Treize étudiants ont qualifié la classe inversée de démarche souple et efficace. Pour la première fois, ils avaient l'impression de prendre leur apprentissage en main. 
- « A l'école comme à l'université, les professeurs nous guidaient. Faites ceci, faites cela. Avec la classe inversée, on a une marge de liberté. On n'apprend pas tous de la même façon. "

- " L'originalité de ce cours est d'apprendre comme on veut. En plus, nous sommes plus proches de la prof. On discute avec elle, on peut lui dire si on n'est pas d'accord sur un point. »

Retour-étudiants

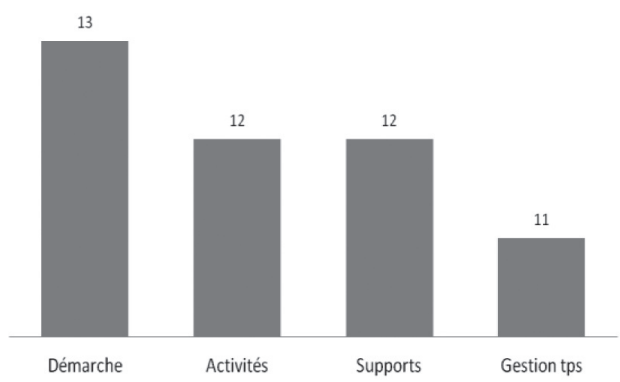

Graphique 1. Qualité- cours

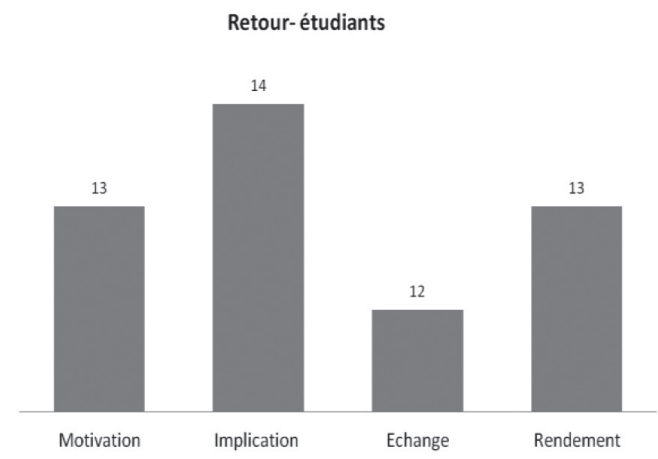

Graphique 2. Engagement

En revanche, trois étudiants ont considéré la classe inversée comme inutile et monotone. Il faut savoir que ces étudiants étaient les meilleurs de la classe et qu'ils ne voyaient pas l'intérêt de sortir de leur zone de confort. Ils avaient aussi le souci d'apprendre vite et bien.

- « On a perdu beaucoup de temps pour apprendre peu de choses. A l'université, le temps presse, chaque minute compte, on n'est pas là pour faire le travail de la prof. »

- « Je n’ai pas aimé cette classe. II ne m’a rien apporté de spécial. Trop de travail et de responsabilités. " 
Douze étudiants ont trouvé les supports motivants et accessibles. Le cours en format vidéo était bénéfique. Par exemple, il a facilité la mémorisation des contenus.

- « Les vidéos étaient accessibles mais un peu longues. Je les regardais tant que je voulais." "

- « Elle donnait les explications sous forme de texte ou de vidéos. C'était facile à comprendre." "

- « J'ai appris à noter les informations avec des schémas. Tout était dans ma tête. »

Le principe du travail collaboratif a intéressé douze étudiants. Ceux-là se voyaient comme des acteurs et des producteurs de leur savoir et non plus de simples consommateurs.

- « Pour la première fois, j'assiste à un cours où je peux faire un vrai travail de groupe. Au début, j'étais un peu perdu. Je doutais de moi-même. Puis, ça a marché. J'étais fier quand madame a demandé à notre groupe de partager notre travail avec les autres. Nos camarades allaient étudier ce que nous avons préparé. ॥

- « J'étais plus à l'aise parce que je savais de quoi le cours allait traiter. Si j'avais une question à poser, je ne me sentais pas seul. Mes camarades pouvaient m'aider. »

- « Je trouve bien de pouvoir poser des questions quand on en a parce que cela nous fait revoir les notions qu'on n'a pas travaillées. »

Sur un autre plan, onze étaient satisfaits de la bonne gestion du temps.

- « Tout était calculé au millimètre. Cette gestion de temps était nécessaire pour ne pas faire du n'importe quoi. »

- « Pas de temps mort. La prof nous mettait la pression. »

Pour les prochains cours, ils ont néanmoins proposé une meilleure gestion des groupes. Le bruit occasionné par les discussions gênait certains étudiants.

- « Tout le monde voulait parler en même temps. La prof a demandé aux étudiants de faire moins de bruit, mais sans résultat. Notre groupe a alors demandé d'aller travailler dans une autre salle. »

\subsection{Retour - enseignante}

Bien que l'on soit tenté par les innovations pédagogiques, il n'est pas facile d'y arriver. Ce n'est pas le cas de Mme MROUEH qui, malgré la surcharge de travail et les difficultés, se félicite des résultats de son expérimentation. 
- « Pour une heure de cours, j'ai fait trois heures de préparation. J'ai tout repensé : la planification des contenus, la création des matériaux pédagogiques, la diversification des activités, l'accompagnement des apprenants. Les étudiants se sont prêtés au jeu alors qu'ils étaient débordés. C'est un public qui travaille le matin, suit des cours de langue l'après-midi et des cours de discipline le soir. »

- " Pour moi le secret de ce succès réside aussi dans l'autonomie que j'ai accordée aux étudiants. Ils étaient volontaires, au lieu de suivre des directives [...] En plus, ils n'étaient pas contraints à rester assis en silence. Ils pouvaient bouger et avoir des échanges riches avec moi et leurs camarades. "

Outre les stratégies d'enseignement-d'apprentissage et l'autonomie, la classe inversée a permis à l'enseignante de développer la mémoire perceptive ${ }^{2}$ (visuelle, auditive, kinesthésique, émotionnelle...) par le biais de supports, d'activités et de modalités de travail axés sur la coopération. Telle que la classe inversée est conçue en étape, il était possible de cibler les différents niveaux de la mémorisation. Passant ainsi de la mémorisation passive à la mémorisation active.

- « Pendant les différentes étapes de la classe inversée, l'apprenant s'implique, joue, met ses sens à l'épreuve, se concentre, s'interroge, vérifie, crée des liens, etc. ce qui booste sa mémorisation et la rend active contrairement à ce qui se pratique dans l'enseignement traditionnel libanais où le par cœur es privilégié. »

- " A chaque étape de la classe inversée, je faisais correspondre un niveau de mémorisation. Arrivé à la fin de l'unité, l'étudiant ne ressentait pas le besoin d'étudier chez lui.»

Par ailleurs, à l'issue de chaque tâche les étudiants rendaient un produit final : l'exposé, la capsule sur le microcrédit, une page HTML sur l'évolution des startups au Liban.

- « Grosso modo, les objectifs pragmatiques et linguistiques sont atteints. Des tâches étaient mieux réussies que d'autres, en fonction des groupes. La capsule était très bien réalisée : le choix des images, les textes courts, l'animation et la musique correspondaient au sujet. Le texte de la page HTML a pris beaucoup de temps. Il y avait des problèmes de reformulation. A propos de l'exposé, les étudiants se sont amusés à se filmer. »

Toutefois, Mme MROUEH n'est pas dupe comme elle le dit :

- « La classe inversée n'est pas la méthode remède à tous les maux. Et il n'est pas question qu'elle devienne le nouveau dogme à la mode, car elle ne convient

\footnotetext{
${ }^{2}$ Il existe cinq types de mémoire : la mémoire de travail (mémoire à court terme située au cœur du réseau), la mémoire sémantique et épisodique (deux systèmes de représentation consciente à long terme), la mémoire procédurale (qui permet des automatismes inconscients) et la mémoire perceptive (liée aux sens).
} 
pas à tout le monde. On peut l'appliquer de temps en temps en fonction des objectifs et des groupes. "

Pour les formations à venir, l'enseignante a préconisé d'aller plus loin avec les étudiants qui ont un bon niveau.

- « A vrai dire, les étudiants faibles ont pleinement profité de la classe inversée. J'étais agréablement surprise par leur motivation. Les étudiants dits brillants s'ennuyaient un peu. Malheureusement, je ne me suis pas assez occupée d'eux. »

\section{Conclusion}

Au terme de cette expérimentation, notre classe inversée a globalement atteint ses objectifs. La mise en place de cette approche sur des bases solides, à partir d'un contrat d'apprentissage clairement défini était incontournable. En effet, les partenaires ont accepté d'inverser les rôles en donnant chacun du sien, selon ses propres moyens.

En dépit des contraintes, l'enseignante a fait preuve d'ingéniosité et de patience pour mener ses étudiants à bon port. A leur tour, les étudiants se sont plu à apprendre dans un esprit de collaboration et de créativité, passant petit à petit d'un apprentissage permissif à un apprentissage constructiviste.

Après avoir énormément investi en temps et en énergie, le produit final (capsules, page HTML, exposé avec Google Slides) était plutôt à la hauteur des attentes. La question reste de savoir si l'expérimentation a abouti parce que tous les acteurs savaient que c'était un essai. Ils faisaient donc l'effort de réussir cette expérimentation.

Quelle que soit la réponse, la classe inversée ne peut se présenter comme la démarche miracle pour lutter contre l'ennui et la passivité des étudiants. L'idée n'est donc pas de substituer une démarche par une autre mais bien de varier les plaisirs, d'alterner au risque de produire le cas inverse. Nous rappelons avec Puren que la classe inversée n'est en elle-même ni une pédagogie, ni une didactique. Par contre, elle est une occasion de relancer les interrogations et les expérimentations pédagogiques et didactiques sur les réponses, forcément plurielles et complexes à apporter aux questions que pose l'enseignement-apprentissage.

\section{Références bibliographiques et sitographiques}

« Apprendre à apprendre : module facultatif. » FMP formation. Web. 2015.

Support de cours. <https://fmpformation.com/Campusapprendre/Support\%20de\%20cours\%20-\%20apprendre\%20a\%20apprendre.pdf>.

Bournaud, Isabelle et Ponsonnet, Geneviève. « Classes inversées : Pourquoi ? 
Quoi ? Comment ? Pour quoi ? » Journée liaison lycées-université 2017 : UFR Sciences. 17 Mai 2015. Ateliers classe inversée.

Blogde M@rcel, un blog sur la pédagogie, la technologie et aussi sur un peu de tout.Web. 13 Juillet 2018. <http://lebrunremy.be/WordPress/>.

Découvrir les concepts de la classe inversée de Eloïse Dufour. Académie Paris. Web. 16 juin 2017. <https://www.ac-paris.fr/portail/jcms/p2_1014285/ decouvrir-les-concepts-de-la-classe-inversee>.

Doceri. "La classe inversée. "Vidéo-clip. YouTube, 2013. 16 avril 2013._https://www.youtube.com/watch?v=1_3_QGPyVCQ>.

Dufour, Héloise. « La classe inversée. » Décryptage Technologie 193. Septembreoctobre (2014). <http://eduscol.education.fr/sti/sites/eduscol.education. fr.sti/files/ressources/techniques/6508/6508-193-p44.pdf>

Dumont, Ariane et Denis Berthiaume. La pédagogie inversée. Bruxelles : Deboeck, 2016. Imprimé.

La classe renversée de David Bouchillon : "Mes élèves produisent du savoir ". Vousnousils l'e-mag de l'éducation. Web. 27 février 2018. <https://www. vousnousils.fr/2018/02/27/classe-renversee-de-david-bouchillon-meseleves-produisent-du-savoir-612568>

Lebrun, Marcel. Théories et méthodes pédagogiques pour enseigner et apprendre: Quelle place pour les TIC dans l'éducation ? Bruxelles : Deboeck, 2007. Imprimé.

Lecoq, Julie et Marcel Lebrun. La classe à l'envers pour apprendre à l'endroit : guide pratique pour débuter en classe inversée. Benoît Raucent - Louvain Learning Lab. Web. 2017. <https://eduq.info/xmlui/bitstream/ handle/11515/35314/lecoq-lebrun-kerpelt-classe-envers-pour-apprendre-endroit-louvain-learning-lab-2017.pdf?sequence=2>.

Le café pédagogique, La classe inversée à l'école de demain. Web. 4 juillet 2016. <http://www.cafepedagogique.net/lexpresso/Pages/2016/07/04072016 Article636032128668325140.aspx>.

Normand, Roy. "La classe inversée : une pédagogie renversante ? " Le tableau 3.1 (2014). <http://pedagogie.uquebec.ca/portail/le-tableau/la-classeinversee-une-pedagogie-renversante-vol3-no-1>.

Mhiri Sellami, Hedia. "La classe inversée avec des adultes en formation continue. " Adjectif. Web. 8 octobre 2018. <http://www.adjectif.net/spip/ spip.php?article478>

Nizet, Isabelle. "La classe inversée : Que peut-elle apportée aux enseignants ? » Canopé. L'agence des usages. Web. 11 février 2015. <https://www.reseau-canope.fr/agence-des-usages/la-classe-inversee-que-peut-elle-apporter-aux-enseignants.html>.

Vahed, Shiva et Mehrabi, Marzieh. « Mise en place de l'approche actionnelle 
dans une classe inversée du FLE : vers une évolution de la pédagogie universitaire. " Francisola, Revue indonisienne de la langue et la littérature françaises 2.1 (2017) : 93-108. Web. 23 juin 2017. <http://ejournal.upi. edu/index.php/FRANCISOLA/article/view/7530/pdf>

\section{THE FLIPPED CLASSROOM AT ISSAE-CNAM OF THE LEBANESE UNIVERSITY: BETWEEN ACTION, REFLECTION AND MEMORIZATION}

This article aims to report on an experiment of the flipped classroom in language learning at the ISSAE-Cnam of the Lebanese University. It was to realize three macro-tasks: a capsule, an HTML page, a presentation via Google Slides, by following three steps: the discovery phase (distance-learning), the analysis and deepening phase (face-to-face and as a group) and the production, evaluation and readjustment phase (face-to-face and distance-learning individually and in groups).The results are conclusive: the students are involved in the project. They became aware of how they learned and carried out tasks in a spirit of sharing and collaboration. These observations, however, do not prevent us from deducing that the flipped classroom is a double-edged sword: it can motivate young people curious about novelties, addicted to new technologies, but it can also be a source of trouble if it is poorly prepared. and misused. Indeed, the investment in energy and time from the protagonists is hardly neglected. Therefore, the conventional class can not be substituted by the flipped classroom, it is the right balance between these two approaches that will make all the difference.

Keywords: flipped classroom, experimentation, collaboration, autonomy, memorization, perceptual memory. 